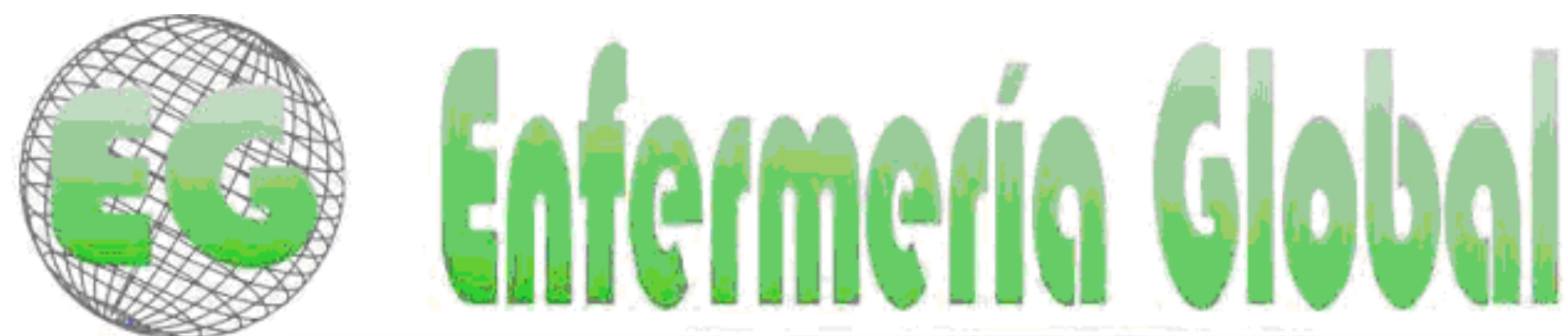

15SN $1696-6$ - $\$$ SS

\title{
LA IMPORTANCIA DEL JUEGO EN EL CONTROL DEL DOLOR PEDIÁTRICO
}

\author{
THE IMPORTANCE OF GAMES IN THE CONTROL OF PEDIATRIC PAIN
}

\begin{abstract}
*García-Carpintero Blas, Eva
*Diplomada en Enfermería. Master Oficial en Ciencias de la Enfermería. Hospital La Paz. Madrid
\end{abstract}

Palabras clave: Dolor, Paciente pediátrico, Juego.

Keywords: Pain, Pediatric Patient, Game.

\section{RESUMEN}

El dolor es un síntoma que acompaña a múltiples enfermedades infantiles, y por consiguiente, motivo frecuente de consulta para los profesionales de la salud. En los niños no fue motivo de preocupación de los cuidadores e investigadores durante mucho tiempo. Sin embargo, la necesidad de analgesia pediátrica ha sufrido un cambio drástico en las últimas décadas, así, el viejo axioma de que los niños no sienten dolor, es afortunadamente un concepto obsoleto. La terapia del juego está comenzando a ser un tema con gran relevancia, ya que puede ayudar a controlar el dolor en el paciente pediátrico y por consiguiente la ansiedad de la familia.

\begin{abstract}
Pain is a symptom that accompanies multiple childhood diseases, and consequently, a frequent motive of consultation for health professionals. In children it was not a motive of worry for the child caregivers or researchers for a long time. Nevertheless, the need for a pediatric analgesia has undergone a drastic change in the last decades. In this way, the old axiom that children do not feel pain is luckily an obsolete concept. Game therapy begins to be a topic with great relevancy, since it can help to control pain in the pediatric patient and consequently the anxiety of the family.
\end{abstract}


El dolor es un problema muy común entre los niños y adolescentes, tal y como han mostrado estudios epidemiológicos recientes (1-3). Tal vez sea esta constatación y la comprobación del impacto que tiene esta experiencia en sus vidas, lo que ha provocado el auge por el interés en el dolor pediátrico. En el año 1980 sólo era posible encontrar 17 artículos sobre el tema, en 1985 eran 30, cifra que se eleva hasta 123 en 1990 y hasta 195 en 1995, y finalmente 262 en 1999 (4). Verdaderamente, los últimos quince años han sido testigos de un aumento espectacular en su estudio en población pediátrica (5).

La mayoría de estos estudios se han orientado a unas condiciones específicas; sin embargo, apenas se ha abordado un análisis extenso de los problemas del dolor pediátrico en general. Estos estudios son importantes a fin de poder tener un mejor conocimiento sobre las características de una enfermedad específica, pero son menos útiles para establecer el cuadro real en la población pediátrica en su conjunto.

La necesidad de analgesia pediátrica ha sufrido un cambio drástico en las últimas décadas, así, el viejo axioma de que los niños no sienten dolor es afortunadamente un concepto obsoleto, comenzando a ser un tema con gran relevancia. En los últimos años asistimos a un incremento del número de trabajos de investigación que tratan de solucionar el problema, atendiendo no sólo al tratamiento, sino también a cómo evaluar su existencia e intensidad, las repercusiones psicológicas del mismo, los mecanismos intrínsecos de su aparición o la atención a los padres (6).

Los niños suponen un alto porcentaje de los pacientes a tratar en los hospitales. La prevalencia del dolor en niños es, al menos, tan elevada como la de los adultos, mientras que la frecuencia de administración de analgésicos es mucho más baja.

Según la Asociación Internacional para el Estudio del dolor, éste se define como "una experiencia subjetiva desagradable que asociamos con lesión mística y que describimos en términos de tal daño o cuya presencia es revelada por manifestaciones visibles $\mathrm{y} / \mathrm{o}$ audibles de la conducta". Esta experiencia emocional desagradable se encuentra a menudo en la actividad diaria de los profesionales de la salud (7). Comprende componentes sensoriales y emocionales, la intensidad de la experiencia y los mecanismos para afrontarlo varían entre los individuos. Los factores ambientales, de desarrollo, de comportamiento, psicológicos, familiares y conductuales repercuten profundamente. Por consiguiente, un mismo tipo de lesión tisular puede causar dolor de distinta naturaleza o intensidad en distintos niños, o al mismo niño en distintos momentos. El entorno material, las actitudes y comportamiento de los cuidadores, así como la conducta, el pensamiento y los estados emocionales, puede acrecentarlo o disminuirlo notablemente.

\section{Importancia del juego en el dolor}

El juego es una actividad propia del ser humano, se presenta en todos los niños, aunque su contenido varíe de acuerdo con las influencias culturales que los distintos grupos sociales ejercen. Los antecedentes del juego se inician en 1928 cuando Freud comenzó a utilizarlo como forma de atraer a los niños a la terapia y como medio terapéutico (8). Tanto Freud como Piaget, consideraron el juego como una actividad valiosa e importante, debido a que sirve como una forma de descarga de tensiones y expresión de sentimientos como la ira, ansiedad o frustración, permitiendo que los niños regulen sus emociones y se comuniquen mediante diferentes conductas, pensamientos o necesidades. Desde entonces, muchos han sido los especialistas encargados de aplicarla en diferentes contextos y empleando diversas 
técnicas y aproximaciones teóricas, pues constituye una alternativa de tratamiento para niños que permite a estos últimos expresarse sin límites.

En la Terapia de Juego, el niño entra a una relación dinámica con el terapeuta que le ayuda a expresar, explorar y encontrar un sentido a sus dificultades, experiencias dolorosas o problemas. Se utiliza para satisfacer las necesidades emocionales de los niños que sufren alguna enfermedad o que se someten a una cirugía y deben ser hospitalizados. La hospitalización es estresante para los niños ya que implica la separación de su entorno familiar y la entrada a un ambiente desconocido(9), con personas extrañas que tocan su cuerpo, hablan con palabras que no entienden y realizan procedimientos que en muchas ocasiones le provocan malestar y/o dolor.

Durante la última década se han incrementado los estudios que revisan las principales técnicas psicológicas (entre ellas el juego) que influyen en la reducción de la ansiedad y dolor de los pacientes pediátricos $(10,11)$. Principalmente se ha utilizado el juego como intervención para ayudar a los niños a superar el estrés generado por una cirugía, la mayoría de los estudios que avalan esta práctica están basados en teorías u observaciones clínicas, aunque la efectividad de estas intervenciones nunca se ha comprobado.

El juego es la ocupación fundamental del niño y por lo tanto es el medio más eficaz para lograr la consecución de cualquier tipo de objetivo de tratamiento en terapia ocupacional pediátrica, puesto que permite aumentar los niveles de desarrollo en los niños, facilitando la adquisición de roles y hábitos ocupacionales, promueve el desarrollo de la adecuada integración sensorial, facilitando la interpretación de la información del medio ambiente (12).

La introducción de terapias alternativas como el juego en el campo asistencial, unidas a la terapia farmacológica pueden provocar beneficios sin ningún tipo de efecto secundario, lo cual influirá directamente tanto en la salud de los niños como en el desarrollo del trabajo diario de las enfermeras en la plantas de pediatría, abordando los cuidados de enfermería de manera holística, incluyendo no sólo el aspecto biológico, sino también el psicológico y social.

\section{BIBLIOGRAFÍA}

1.Groholt EK, Stigum H, Nordhagen R, et al. Recurrente pain in children, socio-economic factors and accumulation in familias. European Journal of Epidemiology 2003; 18: 965-75 2. Kovacs FM, Gestoso M, Gil del Real MT, et al. Risk facors for non-specific low back pain schoolchildren and their parents. Pain 2003; 103:259-68

3. Perquin CW, Hazebroek- Jampschreur. Pain children and adolescents: a common experience. Pain 2000; 87: 51-8

4. Reinosos Barbero, F. El dolor de los niños: ¿un gran desconocido o un gran olvidado? Rev Soc Esp. Dolor. 7:277-278, 2000

5. Fernández- Baena M,García-Peréz A, Ramos M. Importancia del dolor pediátrico en las publicaciones científicas. Rev. Soc. Esp. Dolor 7: 279-284, 2000

6. Ortiz Gordillo E. El dolor pediátrico como motivo de consulta en atención primaria. Vox pediatrica, 10,1 (49-50), 2002

8.Mather L, Mackie J. The incidence of postoperative pain in children. Pain 1983; 15:271-282

7. Internacional Association for the study of Pain,Subcomité on taxonomy pain 1979;6: 249252

Montevideo, 1997.

8. Freud, S.: "Más allá del principio de placer", en Obras Completas, Tomo XVIII, Amorrortu Ed., Bs As, 1984. 
9. Baraibar, R. "Enfoques en pediatría, aspectos psicosociales" Oficina del libro. 10. Moix, J. Preparación psicológica para la cirugía en pediatría. Archivos de pediatría, 47(4): 211-217

11. Cullen k, Mesa PJ, Martínez A, Blanco A. Formulación de un programa de preparación psicológica a la intervención quirúrgica en pediatría. Anal Mod Conduc, 1990;16:209-228.

12. Knox, S.Terapia ocupacional. Madrid España: Panamericana.1998.p18-121 